\title{
Qualitative Study on Health Self-Help Behavior of the Elderly in a Community
}

\author{
Wei LUAN ${ }^{1, \#}$, Yang LIU ${ }^{2, \#}$, An-Li JIANG ${ }^{3, *}$, Ye CHEN ${ }^{1}$ \\ ${ }^{1}$ Renji Hospital Affiliated to School of Medicine of Shanghai Jiao Tong University, Shanghai 201112, China; \\ ${ }^{2}$ School of Nursing, Shanghai Jiao Tong University, Shanghai 200240, China; \\ ${ }^{3}$ Naval Medical University, Shanghai 210000, China
}

\begin{abstract}
Objective: To investigate the current status quo of health self-help behavior of elderly in a community and its influencing factors using a semi-structured interview in a qualitative study.

Methods: A phenomenological method is employed for purposive sampling and snowball sampling methods. The elderly ( $>60$ years old), who had lived in the Minhang District of Shanghai for $>5$ years, with an annual residence duration $\geq 10$ months, were selected. Data were collected in person by a semi-structured and an in-depth interview; the topics were identified according to data arrangement, analysis, and coding.

Results: A total of 10 subjects were enrolled, from Pujiang Town, Minhang District of Shanghai. The cohort constituted of 7 males and 3 females, aged 60-68 years, with an average of (63.40土9.19) years. After data arrangement and analysis, the following topics were obtained: (1) various forms of health self-help behaviors existed among elderly in the community; (2) some factors influenced the health self-help behavior among the elderly in the community; (3) a deviation was observed in the health self-help behavior among the elderly in the community.
\end{abstract}

Conclusion: The health self-help behaviors of the elderly in the community should be rectified to carry out self and the environmental establishment of the health self-help behaviors of the elderly in the community, as well as, to guide and develop the personalized health self-help behavior program.

Keywords: The elderly; Health self-help behavior; Influencing factors

\section{Introduction}

The proportion of the elderly is expected to increase from $6.9 \%$ in 2000 to $19.3 \%$ in 2050 throughout the world ${ }^{[1]}$. After entering old age, the health status of the elderly changes, and the incidence of chronically non-communicable diseases such as hypertension, diabetes as well as cardiovascular and cerebrovascular disease increases ${ }^{[2]}$.

\footnotetext{
"Those authors contributed equally to this work

*Corresponding Author:

An-Li JIANG, Naval Medical University, No. 800, Xiangyin Rd, Shanghai 200433,

China Email: aljiang00@sina.com

Received:April 12 2019; Revised:April 20 2019, Accepted:April 23 2019, Online: May 152019
}

In Germany and other European countries, selfmanagement groups are commonly applied, which can effectively complement the existing social and health services. Presently, there areonly a few studies on the health self-help behaviors of the elderly available in China, which are primarily related to self-help groups and self-help services. A large number of studies have shown that bias is inevitable when employing the questionnairebased survey method for quantitative research. In addition, the overall average condition from a sampling population 
ignores the specific circumstance of the individual; moreover, the quantitative research does not focus on the observer's true feelings. Therefore, some defects in the reporting might occur, and hence, the qualitative study of the health self-help behaviors among the elderly in China is yet rare.

"Self-help" refers to "the act providing help for yourself." In the Modern Chinese Dictionary, the term is defined as "do it yourself" or "self-configuring" (resources) to serve yourself $"$ "[3]. The focus of basic healthcare strategy is that "everyone protects their health" in other countries, and self-help is often considered as one of the elements. At present, there is no universal and authoritative explanation of the concept of health self-help in China. In this study, combining the literature reviews and the above definitions, the operational definition of "health self-help behavior of the elderly" is the activity and self-management of this population to achieve the goal of maintaining health, disease treatment, and rehabilitation.

\section{Materials and Methods}

\section{Study subjects}

Inclusion criteria: (1) Interviewee who had lived in the community of Pujiang Town, Minhang District of Shanghai for $>5$ years, with an annual residence period of $\geq 10$ months; (2) Interviewee whose age was $>60$ years; (3) Interviewee who physically supported interviews, with clear consciousness, was able to communicate in Mandarin or dialect, and was able to communicate with the interviewers; (4) Interviewee with relatively common chronic diseases, excluding some specific, rare, and chronic diseases; (5) All interviewees were willing to participate in the study, and had signed the informed consents, and the families of the interviewees were informed at the same time. Exclusion criteria:(1) Interviewee with mental disorders, cognitive impairment, severe disability, and terminal diseases; (2) Interviewee who was a temporary resident; (3) Interviewee with poor compliance. A total of 10 interviewees were included, and the general data of the interviewees were summarized in Table 1.

\section{Sampling methods}

A combination of purposive sampling and snowball sampling was used. The criteria for the sample size to reach a theoretical saturation were as follows: (1) No new or relevant information to appear in a category; (2) The categories in the data had developed very well, and

Table 1 General information of interviewees $(n=10)$

\begin{tabular}{cccccccc}
\hline No. & Gender & Age (years) & Location & Work & $\begin{array}{c}\text { Education } \\
\text { background }\end{array}$ & Religion & $\begin{array}{c}\text { Family monthly income } \\
\text { (yuan RMB) }\end{array}$ \\
\hline 1 & Male & 66 & Shanghai & Retired & Junior high school & None & $3000-5000$ \\
2 & Male & 60 & Shanghai & Retired & Junior high school & None & $3000-5000$ \\
3 & Male & 61 & Shanghai & Retired & Primary school & None & $3000-5000$ \\
4 & Female & 60 & Shanghai & Retired & High school & None & $3000-5000$ \\
5 & Female & 61 & Shanghai & Retired & Primary school & None & $3000-5000$ \\
6 & Male & 68 & Shanghai & Retired & Junior high school & None & $3000-5000$ \\
7 & Male & 63 & Shanghai & Retired & Junior high school & None & $3000-5000$ \\
8 & Male & 62 & Shanghai & Retired & Primary school & None & $3000-5000$ \\
9 & Female & 67 & Shanghai & Retired & Primary school & Christians & $<2000$ \\
10 & Male & 66 & Shanghai & Retired & Junior high school & None & $<2000$
\end{tabular}


various parts of the model could be closely linked with process and variability; (3) The correlation between the categories could be appropriately established and verified.

\section{Interview methods}

Based on the phenomenological method in qualitative research, data were collected in the face-to-face form, semi-structured interview, and an in-depth interview. The outline of the interview was as follows: What health selfhelp behaviors do you utilize in your daily life? Why do you take this health self-help behavior? Are you willing to take any other health self-help behavior? What are the reasons for unwilling to take other health self-help behaviors? How do you feel about your life after taking health self-help behaviors?

\section{Ethics}

This study was approved by the Ethics Committee of Renji Hospital Affiliated to the School of Medicine of Shanghai Jiao Tong University. All interviewees had signed the informed consent before the interview. The interviewees could refuse the interview at any time. The phone number of the researcher was provided to the interviewees in case any interviewee had a request for interview or health care. All the information of interviewees was strictly confidential.

\section{Data collection and analysis}

The purpose of the present study was explained to interviewees, and interviews were conducted in a quiet place. "I" was used as the research tool, the form of openended question was adopted, and the method of combining statement with simultaneous live recording was utilized. During the interview, the facial expression and behavior of the interviewee is under focus, and the questions of interview and the number should be adjusted timely. The total duration of the interview time was 45-60 min. Upon completion of the interview, the time was recorded, and the number of interviews was 1-2 times. Text data were transcribed verbatim according to the statement and recording materials on the spot. Based on the Colaizzi's seven-step method, the meaningful parts of the text were identified, recurring views were encoded, and coded views were summarized. Subsequently, a detailed description was written, similar views were distinguished, and finally, the transcript returned to the interviewees to confirm the responses in order to ensure the accuracy of the results.

\section{Data analysis}

The collected data were analyzed using Colaizzi's sevenstep method and NVivo8.0 software (Ahead, Germany), and the information was compared and understood, coding was established, and topics identified.

\section{Integrity}

The interviewees were selected through purposeful selection; the elderly individuals who fulfilled the inclusion criteria were selected. Moreover, during data collection, the researcher would be self-reflective and communicate with the research team to identify research topics in order to confirm the integrity of the study.

\section{Results}

\section{Topic 1 Health self-help behaviors}

Various forms of health self-help behaviors were observed among the elderly in the community. (1) Health knowledge acquisition behavior (internet, newspapers, participation in health lectures, consultation with professionals, obtaining health promotion materials, peer communication). Interviewee 1 "If there is a health lecture, I would like to listen to it. Occasionally, I organize health lectures for the members of our association (cycling sports association of Pujiang)." Interviewee 1 "I know a doctor in a health center, and one of my relatives is a doctor too; I often ask them what medicine should I take." Interviewee 4 "I often read newspapers that are ordered by my unit, such as Jian Kang Bao (Health News) and Liberation Daily, and I always pay attention to my health". (2) Health maintenance behavior (choosing the right diet, weight control, making an exercise plan, seeking help from friends, and good habit of work and rest). Interviewee 1 "I go to bed early at 9:30 pm and wake up at 6 am." Interviewee 4 "I drink a cup of plain boiled water in the morning and a yogurt at night." Interviewee 2 "I think it is good for me to do something after retirement. I need to get some work and do some exercises." Interviewee 9 "Sometimes when I am working, I feel 
tired immediately. I occasionally feel lonely, so I often go out for taking a walk with my sister or participating in matchmaking activities." (3) Medical resources utilization behavior (minor illness into community, serious illness into hospitals, participation in public health services, cooperation with physician follow-up, and utilization of mobile medical resources). Interviewee 1 "If I am sick, I will go to a health center and a nearby small hospital. If I take a physical examination, my blood, heart, stool, and urine should be examined. I have undergone physical examination three times." Interviewee 6 "If my condition is serious, I will go to big hospitals, because they are good at everything."

\section{Topic 2 Influencing factors of health self-help behavior among the elderly in the community}

Influencing factors of health self-help behavior among the elderly in the community were as follows: (1) Socio-demographic factors (age, economic income, the degree of education, social stratum, occupation in the past). Interviewee 1 "If milk shelf-life has expired, I still drink it, because I think although it has expired, there is no difference in milk taste; I feel it be wasted if thrown away." Interviewee 5 "In peacetime, I do not pay attention to health TV programs, I do not even read newspapers because I cannot read." Interviewee 6 "I do not eat anything at noon, which is a professional habit that was developed when I worked at a company before. I will have stomach ache and flatulence after eating." Interviewee 7 "I think eye symptoms are related to age and disease, which is normal." Interviewee 9 "Elderly in the countryside often have some problems in the body, I believe that I would get better if I bear it, so I do not go to hospitals often." (2) Psychological factor. Interviewee 6 "My health. Every morning, I wake up, stretch, and feel very comfortable, then I think about what I am going to do today, which makes me feel very cheerful and happy, so I feel very healthy. If I wake up in the morning and feel uncomfortable, such as headache, I feel unhealthy on that day. Moreover, if I feel I am in great spirits today, I will eat more and go somewhere; if I feel very good when I wake up in the morning, I will do morning exercises. When I do not feel any discomfort but very happy, I will think about what I am going to do today, such as playing cards with friends for 1-2 h. I will think about what to do first and what to do next, if I have more time, I will play with my friends, and that would be great." (3) Other prompting factors (interpersonal relationship, emotional state, habits, the influence of family or friends, hobby and interest, the degree of confidence in doctors, and medical condition). Interviewee 1 "Our cycling team has professional mountain bikes and sports equipment." Interviewee 4 "After retirement, I still go to work, it is boring to stay at home, and it is joyful to work outside." Interviewee 5 "My father feels that health is more important when he is sick." Interviewee 5 "I have not been informed to take a physical examination because of my current age; however, if there is a notice, I am willing to take it." Interviewee 7 "I have not played Mah Jong for 2 or 3 years because I have high blood pressure." Interviewee 7 "I have to visit the hospital every week, listen and cooperate with the doctors. I take medicine according to the doctor's advice." Interviewee 9 "I know my friends very well; we often visit each other. Sometimes, I travel with them and play around."

\section{Topic 3 A deviation in the health self-help behavior among the elderly in the community}

A deviations in the health self-help behavior is observed among the elderly in the community. Deviation 1: Different understanding of health. Interviewee 1 "Good health, good mood. I will be healthy as long as I feel happy." Interviewee 6 "My health. I wake up, stretch, and feel very comfortable in the morning, then I think about what I am going to do today, which makes me feel very cheerful and happy, so I feel very healthy." Interviewee 9 "Although there is nothing wrong with my body, I often go to travel and play around; therefore, my mood will be better." Deviation 2: In terms of diet, many elderly people, who were interviewed, lack the basic medical and health knowledge; their understanding of diabetes mellitus remained in the "no sugar" phase. Interviewee 4 "Do not put sugar while cooking, eat less sugar, and do not drink at all." Interviewee 8 "I usually require a strong taste in food, and I add plenty of salt while cooking." Deviation 3: In terms of sports, the elderly interviewed did not know sufficiently about sports. Interviewee 2 "I think working is a kind of sport, and going to work is also a kind of sport." Interviewee 4 "I go to fields to 
do farm work when I am free. I am usually very busy and do not have time to exercise, and I do not have any specific hobby in sports." Interviewee 5 "I do housework, laundry, and cooking as a sport." Deviation 4: In terms of medical treatment, the elderly interviewed believed that it was normal to fall sick. If they had minor diseases, they do not seek immediate medical treatment and choose to wait for some time. Interviewee 1 "Stomach disease is chronic, I will get better after taking medicine, so I do not need to see a doctor; I always take medicine when I feel uncomfortable. I do not pay much attention to health education." Interviewee 2 "When I get cold, I do not take any medicine; I believe that I will be fine after a week."

\section{Discussion}

In the process of active participation and self-management to achieve the purpose of health maintenance, disease treatment and rehabilitation, the elderly in the community have a variety of experiences coexist, but there are few studies on their health self-help behavior at present, and further research and exploration on the health self-help behavior of the elderly by medical workers are needed to improve the above situation.

\section{There are many types of elderly health self- help behavior}

The results of this study show that the elderly in the community have various forms of health self-help behavior, such as health knowledge acquisition, health maintenance behavior and medical resource utilization behavior, but due to the influence of demographic factors, self-perception factors and other psychological factors, there are certain behavioral deviations. The "getting old before getting rich" status of China's aging makes the society (including material and system) enter into the aging process far from being ready. The social old-age care system is under great pressure, and the contradiction between supply and demand of old-age care resources is also very prominent ${ }^{[4]}$. On the basis of "healthy aging", "active aging" is expressed in a broader sense. It enables the elderly to realize their potential in physical, social and spiritual aspects in their life and participate in social activities according to their needs, desires and abilities $^{[5-6]}$. The survey results show that the health selfhelp behavior of the elderly in the community can be affected and controlled by external factors, suggesting that we can build a healthy self-help environment through regulation and construction. Moreover, attention should be paid to the elderly people's psychology ${ }^{[7]}$. Li et al.' ${ }^{[8]}$ and Cao et al' ${ }^{[9]}$ results show that the elderly is higher demand for health check-up, now for the elderly health management service, help to change their lifestyle, and reduce the prevalence of chronic diseases, to promote the elderly health self-help behavior and diversify, eventually reaching a far-reaching influence on their quality of life and health outcomes ${ }^{[10]}$.

\section{The elderly health self-help behavior needs reasonable support and scientific guidance}

The results show that the elderly health self-help behavior is related to social demographic factors and perceived disease factors. At the same time, there were behavioral biases in health perceptions, diet and exercise. The mechanism of influencing factors is not clear. Suggestions based on the theory of "behaviorism psychology"[11] and" health behavior change theory" ${ }^{\text {"[12] }}$ research, can build on health as the center, community and family as the unit, the elderly health self-help demand-oriented "healthy community", and the 3A-level general hospitals -grassroots health institutions-community-family of four, the integration of the whole life cycle health services provide fully available, accessibility of health resources, to the community elderly health self-help behavior can assign ${ }^{[13]}$. "Internet + " for the community elderly health management power, on the one hand, according to the elderly health records for data analysis, setting up risk warning and slow disease prevention and control of management strategy, on the one hand can make the elderly through the equipment and health management system timely contact with professional health management personnel, professional guidance, promote and stimulate the elderly health self-help behavior, rectify the health behavior deviation ${ }^{[14-15]}$.

\section{Summary}

The present study revealed various forms of health self- 
help behaviors among the elderly in a community. Due to the deviation of the health self-help behaviors of the elderly in a community, the theories of behaviorist psychology and behavioral health change in the elderly are combined. The health self-help behaviors of the elderly in the community are rectified; as well as, self and environmental construction and guidance were carried out, which develops a personalized health selfhelp program for the elderly in the community. "Health self-help behavior" is a relatively new concept, which is not yet clearly defined. This study is only a preliminary investigation in this field, and additional studies are essential in the future.

\section{Declaration}

The authors of this article declare they have no conflict of interest.

\section{References}

1 Gavrilov LA HP. Aging of Population. New York: McMillan, 2006.

2 Sun SY, Zeng H, Zhang LP. Population Ageing and Nursing Present Conditions and Countermeasures in China. J Nurs Sci, 2006, 21(21): 76-78.

3 Borgetto B, von dem Knesebeck O. Patient selfhelp, user perspective and health services research. Bundesgesundheitsblatt, Gesundheitsforschung, Gesundheitsschutz, 2009, 52(1): 21-29.

4 Qiu Yang. Research on the impact of aging population on medical expenditure in China. Popul Soc, 2015, 31(2): 95-102.

5 General Report Drafting Group, Li zhihong. General report of national strategy research on population aging. Sci Res Aging, 2015, 3(3): 4-38.

6 Song Quancheng, Cui Ruining. Theoretical response to rapid aging of population-from healthy aging to active aging. Shandong Soc Sci, 2013(4): 36-41.

7 Hodges JQ, Markward M, Keele C, et al. Use of self-help services and consumer satisfaction with professional mental health services. Psychiatr Serv, 2003, 54(8): 1161-1163.

8 Li jun, Wang Deliang, Wang Jiaji, et al. Cognition and demand intention of health management of middleaged and elderly farmers in mountainous rural areas and its influencing factors. Chin J Dis Contr Prev, 2013, 17(5): 429-432.

9 Cao haitao, Pan Yihui, Yu Xiaojing, et al. Research on the cognition and demand status of health management services for residents in zhabei district. Shanghai Chin Gen Pract, 2013, 11(10): 1606-1608.

10 Shi FF, Li L, Liu ZJ, et al. Qualitative assessment of health management service needs of elderly residents in urban communities-a case study of chengdu. J Prevent Med Inform, 2014, 30(2): 101-104.

11 Liu Yifeng. A brief analysis of the basic principles of behaviorism psychology and its influence on clinical psychology. Chin Med, 2013, 26(5): 988-991.

$12 \mathrm{Xu}$ Yongneng, Lu Shaoping, Lin Jianhua, et al. Application of health behavior change integration theory to improve the rehabilitation effect of homebased elderly bedreds after discharge. China Nurs Manag, 2015, 15(7): 786-789.

13 Wu Zhijun, Jian Weiyan. Study on availability and social determinants of community health management services for elderly patients with chronic diseases. Chin Gen Med, 2015, 18(28): 3469-3472.

14 Li Xiaonan, Sun Junfei, Ni Xiaoling, et al. Discussion on Internet + community health management service model. Chin J Health Inform Manag, 2016, 13(1): 8588.

15 Liu Hanyang, Mu Yunqing, Feng Zeyong. Enlightenment of American managed medicine on community health management in China. Med Philos(A), 2015, 36(9): 74-77. 